\title{
DEMIGRATION - A BETTER WAY TO DERIVE AN INTERPRETATION OF UNMIGRATED REFLECTIONS
}

One key to model-based depth imaging procedures is the ability to work flexibly in the three principle seismic domains; migrated time, unmigrated time and depth. The observations and analyses performed in each of these domains play a distinct role in creating an earth model accurate and robust enough to yield a suitable depth image for exploring in the complex and subtle structure environment.

Surfaces defined within the depth domain describe the earth model used for migration. These surfaces must be derived from observations made in either the time-migrated or unmigrated time domains. The choice is important. Observations made in the unmigrated time domain provide the most accurate basis for defining surface structure in depth (using inverse normal incidence rays), because they assume less about the data condition. However, because the unmigrated image looks less like an image of the geologic structure, its interpretation is often more difficult to make.

In contrast, the time-migrated section provides a closer rendering of geologic structure, greatly facilitating interpretation. Interpreted horizons can be transformed to depth using the inverse image ray. The drawback in interpreting time-migrated reflections is the diminished accuracy due to the added assumptions related to data condition. These assumptions are (1) the outcome of the time migration procedures employed is properly represented by the image ray, (2) the velocity field employed in the timemigration is accurate, and (3) when creating reflection maps from 2D-migrated grids, only those portions of reflections in dip orientation are used. In the complex structure environments these assumptions are often violated to a significant degree.

We present a method which allows the user to experience the advantages of both approaches (unmigrated and time-migrated interpretation) but the disadvantages of neither; a method that utilizes observations made in the time-migration domain but derives depth structure from unmigrated reflections. The necessary link is demigration, the transformation of time-migration interpretation to the unmigrated domain.

Demigration is the inverse of time-migration, operating on reflection interpretations rather than amplitudes. The output of demigration is a predictor of and guide to unmigrated reflection geometry. The sequence of operations is (1) time-migrate the stack with simple velocity model, (2) interpret the time-migrated reflections, (3) demigrate the interpretation with an operator that is the direct inverse of the timemigration, using the identical velocity model employed in the time-migration, (4) examine the result to confirm conformity with unmigrated reflections, and modify if necessary, and (5) inverse-normal-incidence ray-trace the unmigrated reflections with optimum velocity model to derive the final structure. 
It is important to recognize the limited role played by the time-migration in this process, as compared to its conventional use. Here, it is used only to create an image reliable enough to ascertain the structural nature of reflections, (for example, is a reflection from the top-of-salt or base-of-salt), with no pretense of image accuracy. Its role is solely to provide a better pathway for interpreting reflections on the unmigrated section.

It is because of this more limited role that this process avoids the errors attendant with the assumptions listed above. For example, because the time-migrated interpretation is not directly taken to depth, the inverse image ray is not employed. One need not be concerned about whether the outcome of the time-migration procedure used is actually represented by the image ray. Also, errors in the final structural model do not result from errors in the simple velocity model because this model is used for both timemigration and demigration. Similarly, errors in the final structural model do not result from sideswipe as long as sideswipe is not so severe that it renders reflections unintelligible after time-migration.

Examples of demigration are shown in both two and three dimensions. Of particular concern is the ability to derive three-dimensional multivalued reflections in the unmigrated domain from single valued reflections in the time-migrated domain.

\section{Imaging and Modeling Data Flow}
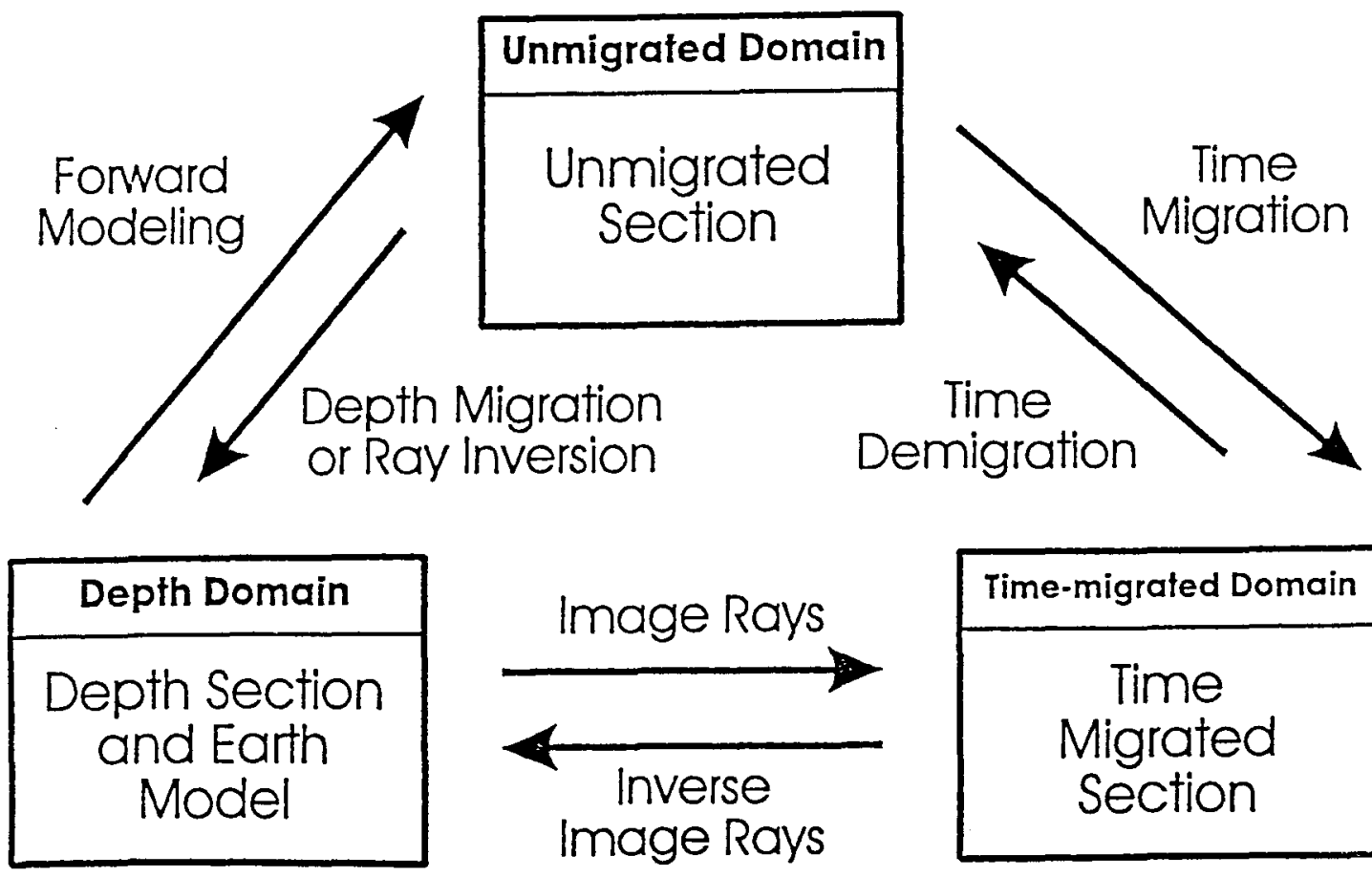

Time-migrated Domain

Time

Migrated

Section 